\title{
Avaliação de modelos obtidos por diferentes sistemas de digitalização 3D para produtos personalizados
}

Evaluation of models obtained by different 3D scanning systems for custom products

Clariana F. Brendler ${ }^{1}$

Fábio P. da Silva ${ }^{2}$

Fábio G. Teixeira ${ }^{3}$ 


\section{Resumo}

O objetivo é avaliar modelos obtidos por diferentes sistemas de digitalização tridimensional e verificar se as variações geométricas podem gerar implicações nas medidas antropométricas para o projeto de produtos personalizados. Para as comparações e análises, a perna direita de um sujeito foi digitalizada, pelo scanner 3D de luz branca (Artec Eva) e pelo scanner por infravermelho (kinect da Microsoft). Os modelos 3D obtidos foram comparados através do software Geomagic Qualify. Também, através da usinagem CNC foram realizadas comparações de análises visuais, por mapeamento de pressão e por termografia. Os resultados obtidos foram satisfatórios tanto para o uso do scanner Kinect quanto para o Artec Eva. Porém, houve uma diferença dimensional de aproximadamente $7 \mathrm{~mm}$ entre os modelos 3D, o que demonstra uma maior precisão de medidas nos modelos obtidos pelo scanner Artec Eva. No entanto, não invalida o uso do Kinect para o desenvolvimento de projetos de produto personalizados. O que irá determinar qual o scanner 3D mais adequado para cada tipo de produto é a necessidade do grau de precisão para que o produto desempenhe sua função com maior conforto e eficiência.

Palavras-Chave: Digitalização 3D, projeto de produto, modelos, medidas antropométricas.
The purpose is to evaluate models obtained by different 3D scanning systems, and that the geometric variations can generate implications for anthropometric measurements for custom product design. For comparison and analysis, the right leg of a subject was scanned by white light 3D scanner (Artec Eva) and the infrared scanner (Microsoft Kinect). 3D models obtained by the scanners were compared using the Geomagic Qualify software. Also, by CNC machining of visual comparisons were performed analysis, for pressure mapping and thermography. The results were satisfactory both for the use of Kinect scanner and for Artec Eve. However, there was a dimensional difference of about $7 \mathrm{~mm}$ between the 3D models, which shows higher accuracy measures the models obtained by Eve Artec scanner. In however, does not invalidate the use of Kinect for developing custom product designs. What will determine the most suitable for each type 3D scanner product is the degree of accuracy necessary for the product to perform its function with greater comfort and efficiency.

Keywords: 3D scanning, product design, models, anthropometric measurements.

ISSN: 1808-3129

\footnotetext{
1 Universidade Federal do Rio Grande do Sul - UFRGS Porto Alegre, RS, Brasil clafischer@hotmail.com ${ }^{2}$ Universidade Federal do Rio Grande do Sul - UFRGS Porto Alegre, RS, Brasil fabio.silva@ufrgs.br
}

\author{
${ }^{3}$ Universidade Federal do Rio Grande do Sul - UFRGS \\ Porto Alegre, RS, Brasil \\ fabiogt@ufrgs.br
}

Artigo indicado através da organização do evento IDEMi 2015 


\section{INTRODUÇÃO}

Para o desenvolvimento de projeto de produtos personalizados, como os produtos para o vestuário, calçados e tecnologia assistiva (TA), o ideal é obter as medidas antropométricas diretamente no usuário final. Um produto melhor adaptado à anatomia do usuário significa maior conforto, menos risco de acidente e melhor desempenho de sua função. A precisão das medidas antropométricas, portanto, está diretamente associada à função e às especificações dos produtos (ULRICH; EPPINGER, 2008; IIDA, 2005; LU et al., 2010).

A função do produto em relação ao seu uso pode ser classificada em função prática, estética e simbólica, na qual sempre uma destas funções se sobressai no desenvolvimento do projeto de produto (LÖBACH, 2000).

No caso da função prática são valorizadas as necessidades fisiológicas do usuário. Desta forma, a precisão das medidas antropométricas tem especial importância para uma maior função ergonômica do produto e, assim, maior desempenho das necessidades físicas do usuário (CLARKSON,2008; LÖBACH, 2000).

Para o desenvolvimento do projeto de produto, do ponto de vista industrial, o ideal seria fabricar um único tipo de produto padronizado, pois isso reduziria os custos. Contudo, para os usuários e/ou consumidores, isso nem sempre proporciona conforto e segurança. A adaptação do produto ao usuário se torna mais crítica no caso dos produtos de uso individual como os personalizados (IIDA, 2005).

Para o desenvolvimento de produtos personalizados, o uso do modelo do processo de Design Centrado no Usuário (UCD) é particularmente o mais apropriado. No processo de UCD há o envolvimento do usuário em todas as etapas do processo de projeto (WANG, 2009).

O Design Centrado no Usuário é um processo importante para o desenvolvimento de produtos personalizados, uma vez que os designers terão requisitos mais precisos em relação ao usuário final do produto. Neste processo, a primeira etapa compreende o estudo sobre o usuário e o produto que irá utilizar (HERSH, 2010).

Na segunda etapa, usa-se o conhecimento das necessidades do usuário para os requisitos e restrições do projeto. A seguir, na terceira etapa são apresentados protótipos ao usuário para avaliação e, na última etapa, é reavaliado o projeto conforme as questões identificadas nas etapas anteriores.

Realiza-se, um ciclo interativo de teste, design, medidas antropométricas e redesign. Assim, conforme o modelo de design centrado no usuário as medidas antropométricas são fundamentais para o desenvolvimento de projeto de produto, principalmente se tratando de produtos personalizados (HERSH, 2010). As medidas antropométricas podem ser obtidas por métodos diretos (manuais) ou por métodos indiretos (digitalizadores 3D).

O levantamento das medidas antropométricas por meio de medições manuais não fornecem dados precisos e confiáveis, principalmente, relacionados a forma e silhueta do corpo. Muitas vezes, dependendo da anatomia, há medidas que são impossíveis de serem obtidas pelo método manual (LU; WANG, 2008; SIMMONS, 2001; TOMKINSON; SHAW, 2013).

Com o avanço da tecnologia, pesquisas vêm sendo realizadas para a obten- 
ção de dados antropométricos por meio de digitalizadores tridimensionais para que seja possível a obtenção dos dados antropométricos mais precisos e confiáveis (SIMMONS, 2001; TOMKINSON; SHAW, 2013; WANG et al.,2007).

Assim, a digitalização tridimensional possibilita capturar dados da superfície dos membros dos usuários sem o contato físico para, posteriormente, desenvolver um modelo 3D virtual. A partir do modelo, o projeto de um produto personalizado pode ser desenvolvido de acordo com a característica física de cada usuário. A aplicação da digitalização 3D como uma ferramenta para auxiliar no desenvolvimento de projeto de produto, principalmente, no âmbito das medidas antropométricas e obtenção de modelos 3D do corpo para o desenvolvimento de produtos personalizados, é incipiente e carece de mais estudos na área.

Referente à estudos de comparação e precisão dos modelos 3D entre os diferentes tipos de scanners 3D, há pesquisas relevantes como dos autores: Foure et al. (2011), Li et al. (2008), Ozsoy et al. (2009) e Han et al. (32010). Porém, estas pesquisas não relacionam a digitalização 3D com o desenvolvimento de projeto de produto. Nestes estudos, foram observadas vantagens e desvantagens entre os diferentes sistemas de digitalização tridimensional e sistemas manuais de medição antropométrica. São abordadas questões sobre a influência da iluminação do ambiente no momento da varredura da imagem, problemas de precisão relacionados à medição manual do corpo humano e a influência do movimento do corpo na geração do modelo 3D durante a digitalização 3D.

As análises de medidas antropométricas e precisão são possíveis através da comparação entre os modelos 3D obtidos por diferentes sistemas e métodos de digitalização tridimensional. Estas comparações são possíveis, por exemplo, através de métodos como a sobreposição dos modelos 3D analisados em softwares específicos para este fim. Também, por simulações de uso de um produto desenvolvido utilizando os modelos 3D como referência para obtenção das medidas antropométricas e avaliados através de análises visuais, termografia e mapeamento de pressão.

Por meio das análises por mapeamento de pressão é possível verificar o local onde há maior conforto ou desconforto em relação ao uso de um produto pelo usuário. Através da termografia, é possível analisar onde as regiões recebem maiores temperaturas após o uso de um produto e, nas análises visuais, é possível visualizar precisão de encaixe, forma e, consequentemente, o conforto de um produto.

Portanto, o objetivo deste artigo é avaliar modelos obtidos por diferentes sistemas de digitalização 3D. Para isso, foi realizada uma comparação entre os modelos tridimensionais obtidos pelos scanners Artec Eva e Kinect. Foi avaliada a precisão dimensional e suas possíveis implicações no desenvolvimento de projetos de produto personalizados.

\section{ANTROPOMETRIA NO DESENVOLVIMENTO DE PRODUTOS}

Ergonomia já foi definida como "tecnologia de projeto" baseando-se em ciências biológicas como anatomia, fisiologia e psicologia. Também, definem em outro momento que "ergonomia é simplesmente uma ciência interdisciplinar que estuda as 
relações entre as pessoas e seus ambientes" (PANERO; ZELNIK, 2002).

A ergonomia parte do conhecimento do homem para fazer o projeto do produto ajustando-o às suas capacidades e limitações. Portanto, estuda as características do usuário para, depois, projetar o produto (IIDA, 2005).

O Design determina métodos para desenvolvimento de produtos e a Ergonomia ajusta estes produtos às necessidades e às capacidades humanas. Portanto, são disciplinas científicas que compreendem a relação homem $X$ tecnologia (PASCHOARELLI, 1997).

A ergonomia está sendo trabalhada cada vez mais em domínio especializado, dividindo-se em três as características específicas a serem abordadas. São elas: a ergonomia física, a cognitiva e a organizacional (IIDA, 2005). Na ergonomia física, são tratados os conhecimentos relacionados aos dados antropométricos, precisão, conforto e uso de um produto.

A antropometria passa a ser utilizada como referência em requisito de projeto de produto, definindo dados relevantes para um desenvolvimento de produto (PEQUINI, 2005).

Entretanto, foi apenas em 1950, que a antropometria passou a ser reconhecida como disciplina (SIMMONS, 2001).

A antropometria tem contribuído para a melhoria da qualidade dos produtos de consumo, adaptando-os melhor às necessidades e características do consumidor. Deve-se avaliar para o projeto de um produto, seu público alvo, e considerar a grande variação corporal entre etnias, sexo, idade, fatores socioeconômicos bem como suas particularidades e necessidades em função das limitações e capacidades humanas (IIDA, 2005).

Assim, o desempenho do produto, que está atrelado à sua função pode ser otimizado a partir da consideração dos dados antropométricos corretos. Para isso, é necessária a identificação de quais dados antropométricos serão considerados para cada projeto e, assim, afirmar que a antropometria tem valor fundamental para o Design (SCHOENARDIE et al., 2011).

Não há duas pessoas iguais em todas as suas características mensuráveis e essa singularidade tem sido objeto de estudos e pesquisas há mais de 200 anos (SIMMONS, 2001).

Portanto, é fundamental uma definição correta da população usuária em termos de idade, sexo, etnia e profissão. Se é um produto utilizado por um único usuário, necessitando de personificação nos dados antropométricos, ou se é um produto para uso de um grupo maior de pessoas (CLARKSON, 2008).

O método de obtenção das medidas antropométricas mais adequado para o desenvolvimento de produtos personalizados é por digitalização tridimensional. Uma vez que, a partir da digitalização 3D é possível obter o modelo 3D do corpo do usuário conseguindo medidas de forma, silhueta e volume de partes do corpo que possibilitam dados mais precisos para a personalização de medidas antropométricas (SIMMONS, 2001; BRENDLER, 2013). 


\section{A DIGITALIZAÇÃO 3D}

A digitalização 3D funciona, em geral, por meio de uma varredura medindo a localização dos pontos de uma superfície no espaço ( $\mathrm{X}, \mathrm{Y}$ e Z). Os scanners 3D mais populares, geralmente, trabalham com a técnica de triangulação e, depois de finalizada a varredura do objeto a ser digitalizado, é obtido o mapeamento ponto a ponto da superfície, o qual é denominado nuvem de pontos (SILVA, 2011). Após o processamento dos dados, é gerada uma malha tridimensional que representa um modelo 3D, o qual pode ser reproduzido por equipamentos de usinagem CNC ou impressoras tridimensionais.

Os digitalizadores tridimensionais tradicionalmente utilizados no mercado são por sistema a laser, luz branca ou por fotogrametria (D'APUZZO, N., 2009). O problema é que scanners 3D industriais exigem alto investimento financeiro. Ainda, os scanners a laser ou sistemas por fotogrametria requerem especialização para manipulação dos equipamentos (SILVA, 2011).

O Kinect da Microsoft pode ser considerado uma opção de scanner 3D de baixo custo em relação aos scanners convencionais de digitalização tridimensional no mercado (TONG et al., 2012).

O Kinect foi desenvolvido originalmente para atuar com o videogame Xbox $360^{\circ}$. Este, contém um sensor de movimento e foi desenvolvido para permitir que os usuários interajam com o game sem a necessidade de um controle tradicional ou joystick, pois o sensor reconhece gestos do usuário e comandos de voz. O acionador para o reconhecimento de gestos é um conjunto composto por um emissor e um leitor de infravermelhos. O sistema utiliza a câmera de infravermelhos (IR Depth Sensor) para detectar uma malha de laser projetada sobre os objetos. O Kinect também possui uma câmera de vídeo a cores (Color Sensor) que fornece os dados de cor para o mapa de profundidade (GONZALEZ et al., 2013).

Segundo alguns autores como: Tong et al. (2012); Dutta (2012); Aitpayev e Gaber, (2012) o kinect vem sendo utilizado em animações 3D, auxílio em aplicações de projetos em realidade virtual para espaços físicos e em computação gráfica, no qual requer realismo nos modelos 3D de corpos humanos. No entanto, o principal problema é que o Kinect tem baixa resolução X e Y e precisão de profundidade para a digitalização tridimensional. Para abordar esta questão, há pesquisas sendo realizadas para melhorar a qualidade dos dados e da resolução da profundidade (TONG et al., 2012).

\section{MÉTODOS E PROCESSOS}

Os métodos e processos consistem na análise comparativa de precisão de medidas antropométricas entre os processos de digitalização tridimensional utilizando o scanner Artec Eva (luz branca) e o Kinect (infravermelho). Na figura 01 são apresentadas as etapas para o desenvolvimento da pesquisa.

A primeira etapa consiste na digitalização tridimensional. O scanner Artec Eva possui um custo financeiro maior em relação ao Kinect, com uma relação de, aproxi- 
madamente, 40 vezes. Ambos são sistemas portáteis e fáceis de serem manipulados para a aquisição dos modelos 3D. Os modelos 3D obtidos (digitalizados 3 vezes por cada sistema) foram sobrepostos e exportados para o software Geomagic Qualify, onde foi possível realizar análises de diferença dimensional, forma e volume dos modelos.

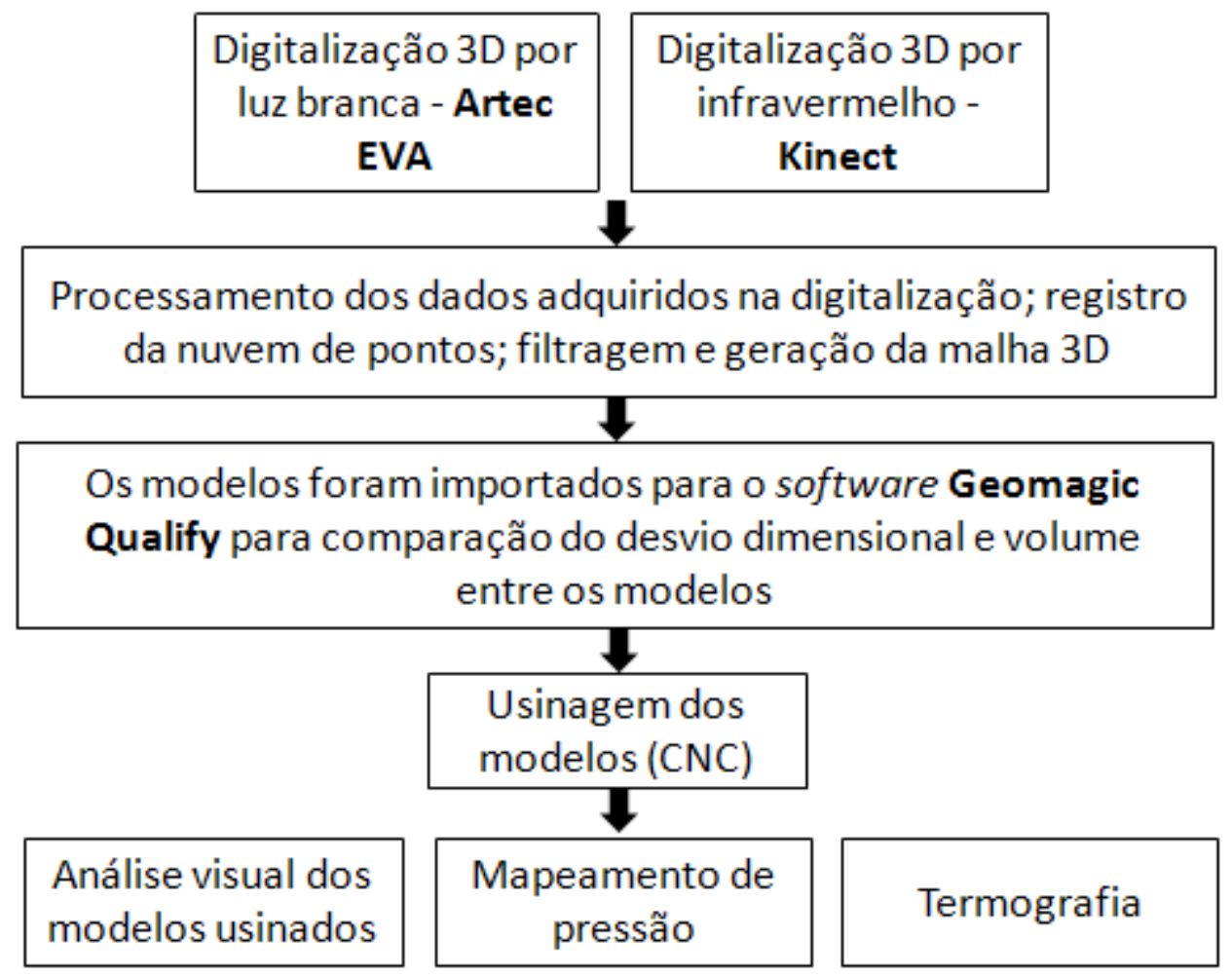

Figura 01: Métodos e processos.

A segunda etapa consiste no processo de usinagem CNC de uma determinada região do modelo 3D obtido pelos processos de digitalização. Para tanto, foi utilizada uma fresadora (Digimill 3D) e um bloco de espuma rígida de poliuretano D40. Foi obtido o molde negativo do modelo para realização das etapas de análise visual, mapeamento de pressão (sistema FSA - Force Sensitive Applications) e termografia (termógrafo Testo T890). Todas as análises foram desenvolvidas no Laboratório de Design e Seleção de Materiais da Universidade Federal do Rio Grande do Sul (LdSM/ UFRGS).

\section{PROCESSO DE DIGITALIZAÇÃO 3D}

Conforme já representado na figura 01, a perna de um sujeito foi digitalizada com o scanner Artec Eva (figura 02). O processo foi repetido três vezes para analisar a coerência e a repetibilidade das malhas tridimensionais dos modelos 3D obtidos por esse processo de digitalização tridimensional. 


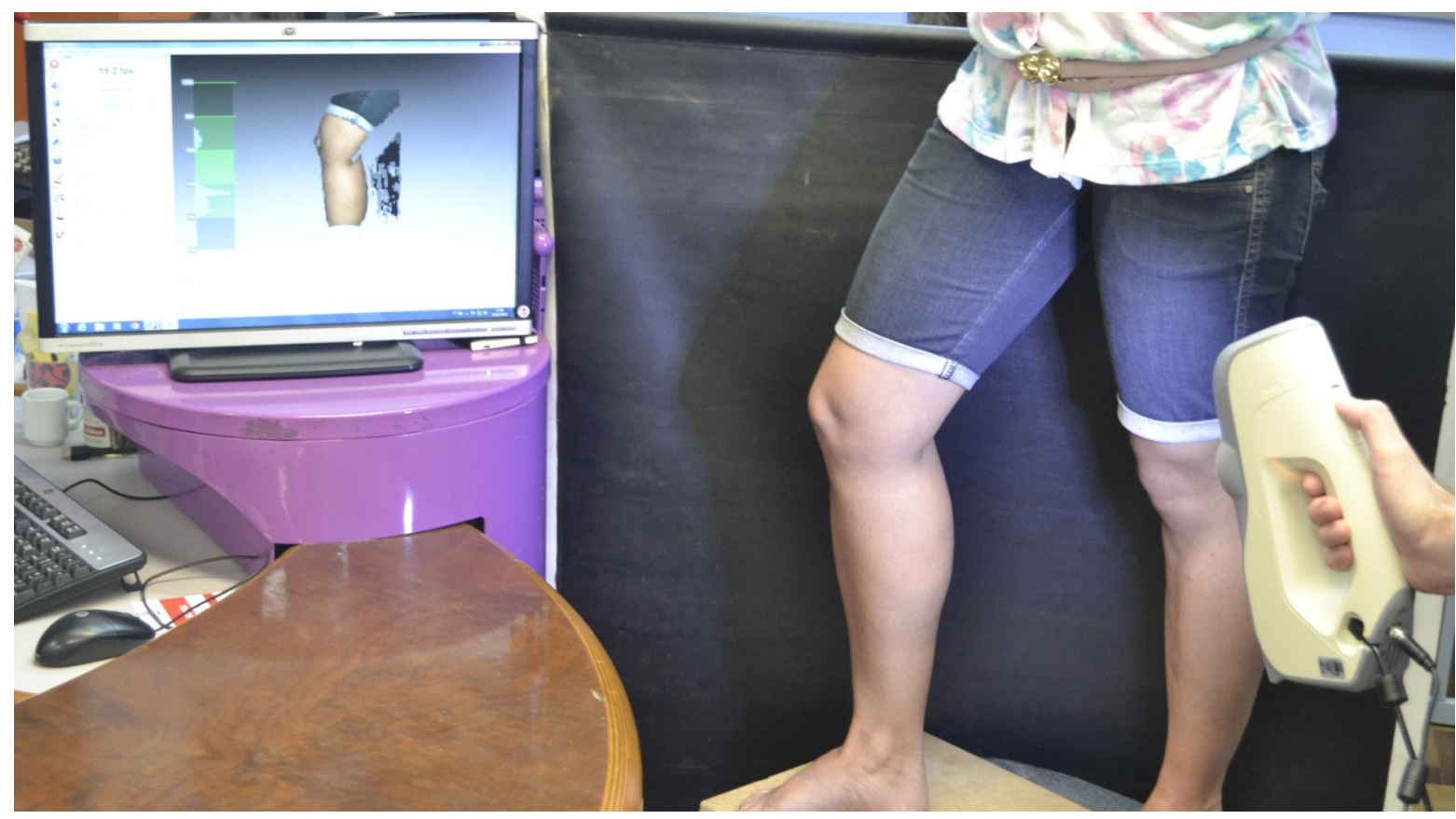

Figura 02: Digitalização com o Scanner de luz branca.

Este processo também foi repetido por três vezes com o scanner Kinect (figura 03). Para cada digitalização realizada, o sujeito permaneceu em uma posição estática por aproximadamente 30 segundos. Após, foi realizado o processamento dos dados adquiridos, o registro das nuvens de pontos, filtragem e geração da malha 3D.

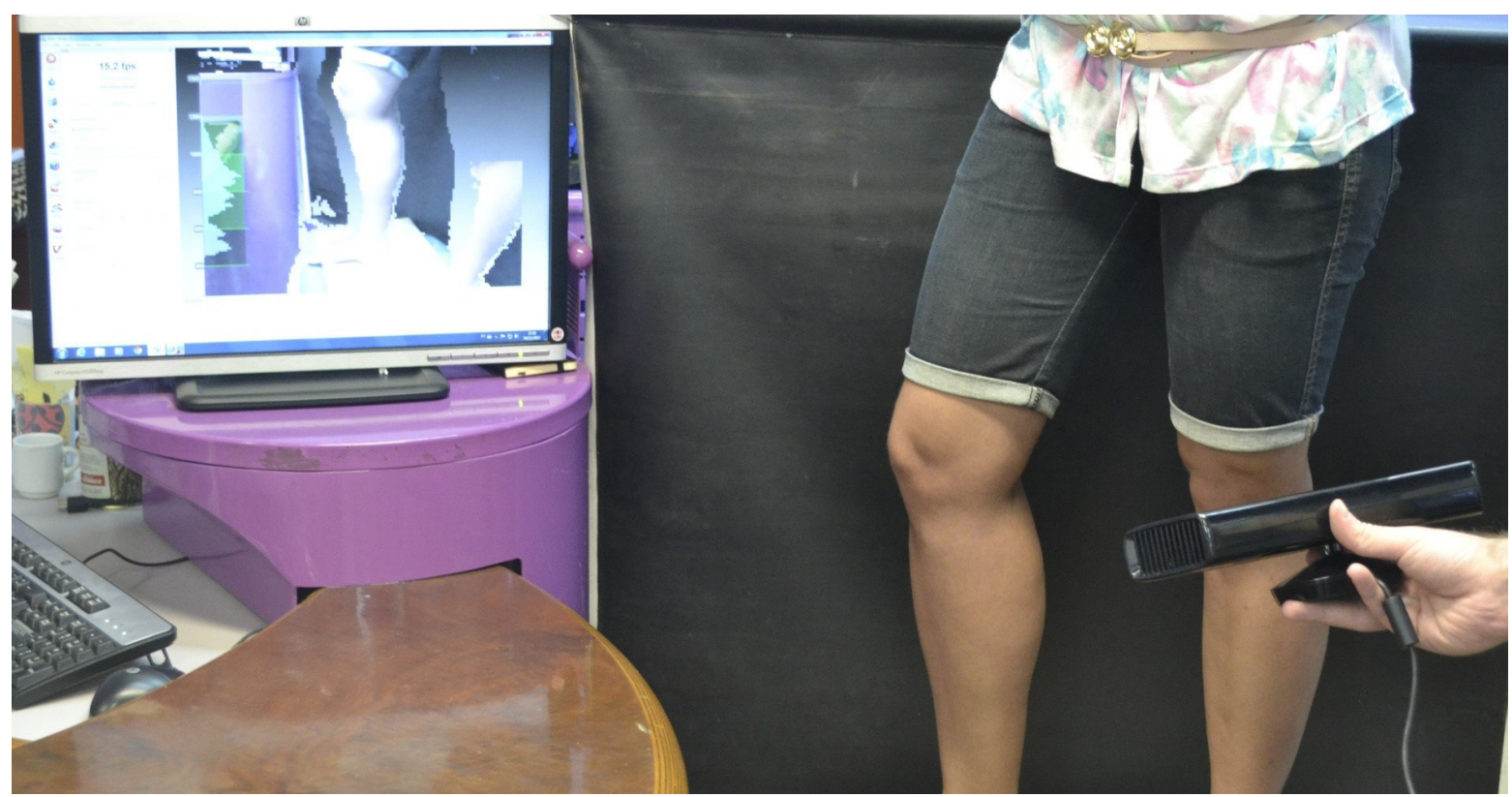

Figura 03: Digitalização com o Scanner Kinect branca.

Os modelos tridimensionais obtidos por cada sistema de digitalização foram sobrepostos entre si e importados para o software Geomagic Qualify onde foram realizadas as análises e comparações dimensionais. A figura 04 apresenta os modelos tridimensionais ( $A$ e B) obtido pelo scanner de luz branca Artec Eva e (C e D) obtido pelo Kinect, vistas anterior ( $A$ e $C$ ) e posterior (B e D) da perna. Nota-se uma diferença visível de precisão, principalmente, nos detalhes dos modelos 3D. 

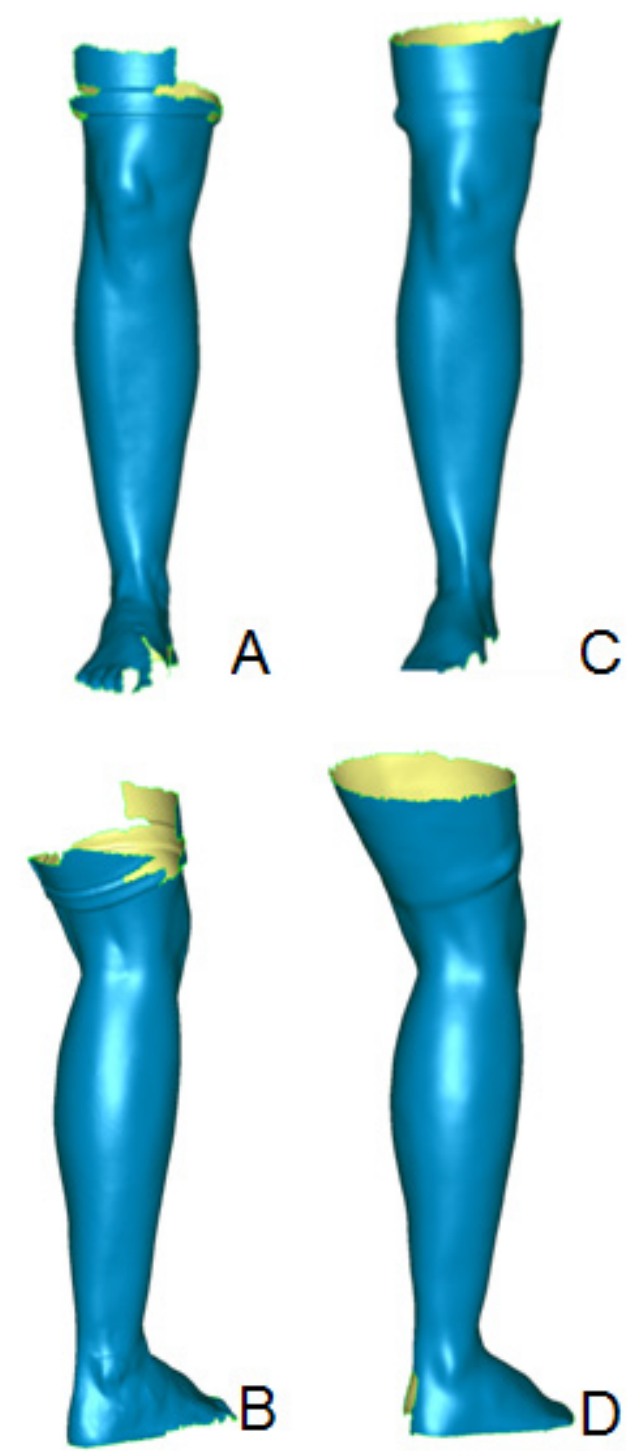

Figura 04: modelos 3D sobrepostos

\section{PROCESSO DE USINAGEM CNC}

Para realizar as análises de contato por mapeamento de pressão e termografia foi necessário desenvolver um molde negativo do modelo 3D da perna digitalizada.

Para as etapas de análise visual, mapeamento de pressão e termografia foi selecionada uma região da perna para obtenção do molde físico negativo pelo processo de usinagem CNC. A figura 05 apresenta a região selecionada para análise, (A) representa o modelo 3D obtido pelo Artec Eva e (B) o modelo 3D obtido pelo Kinect.

Foram desenvolvidos dois moldes negativos, um para cada modelo 3D obtido pelos diferentes sistemas de digitalização, a fim de analisar a precisão no momento do encaixe na perna do sujeito digitalizado. 


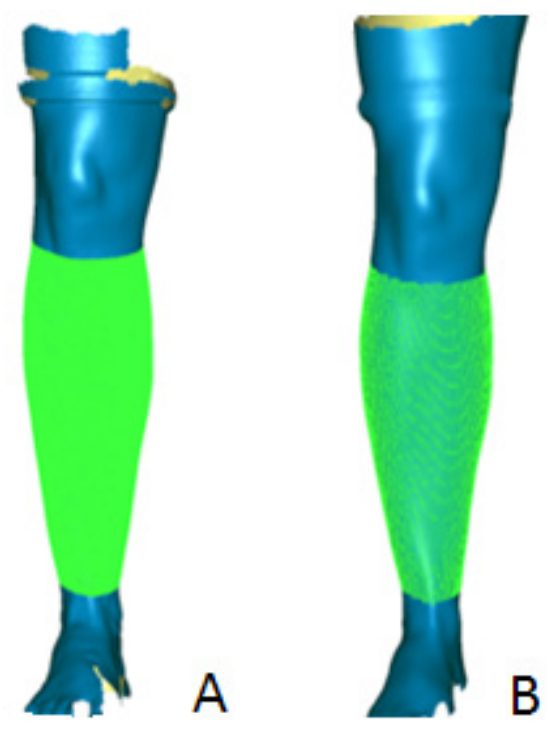

Figura 05: região usinada

A figura 06 apresenta a programação do processo de usinagem CNC sobre uma peça de espuma rígida de poliuretano D40. A usinagem CNC é um processo automatizado e controlado por computador. O equipamento utilizado foi uma fresadora Digimill 3D, na qual se acopla uma ferramenta cortante que faz movimentos de rotação e translação sobre o material a ser processado.

$\mathrm{Na}$ etapa de programação, são planejadas as estratégias de deslocamento da ferramenta e atribuídos os parâmetros de corte para o material a ser usinado. A usinagem foi realizada em duas etapas, uma de desbaste (figura 07) e outra de acabamento (figura 08), ambas com ferramentas de $10 \mathrm{~mm}$ de diâmetro. $O$ processo demorou cerca de 30 minutos.

Após o processo de usinagem, com os moldes negativos finalizados, passou-se para a etapa de realização das análises.

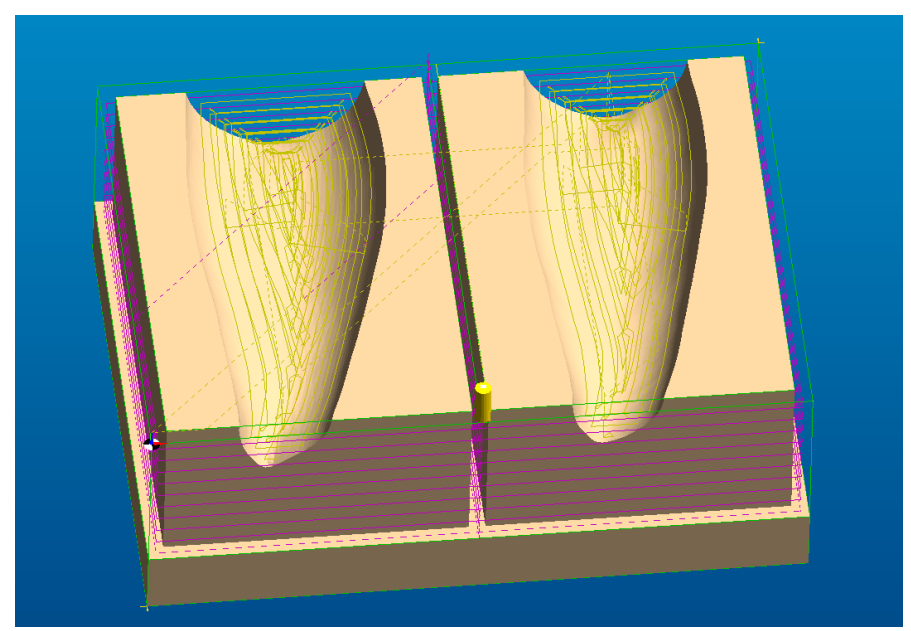

Figura 06: processo de usinagem- programação 


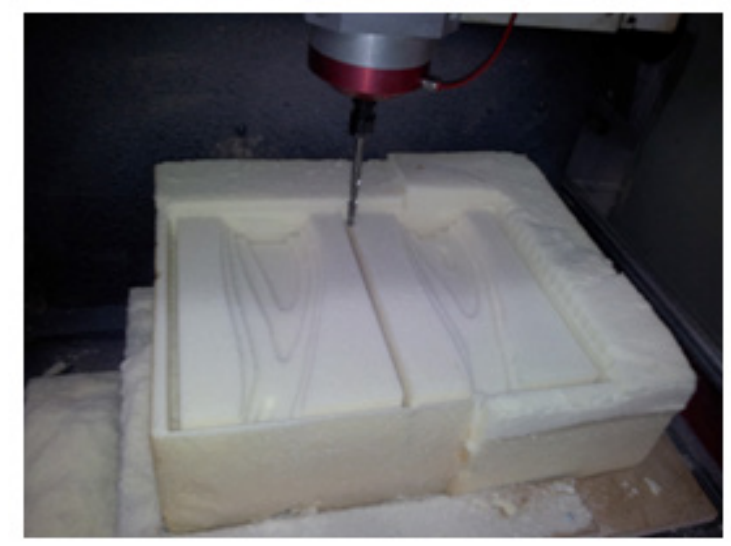

Figura 07: usinagem - desbaste

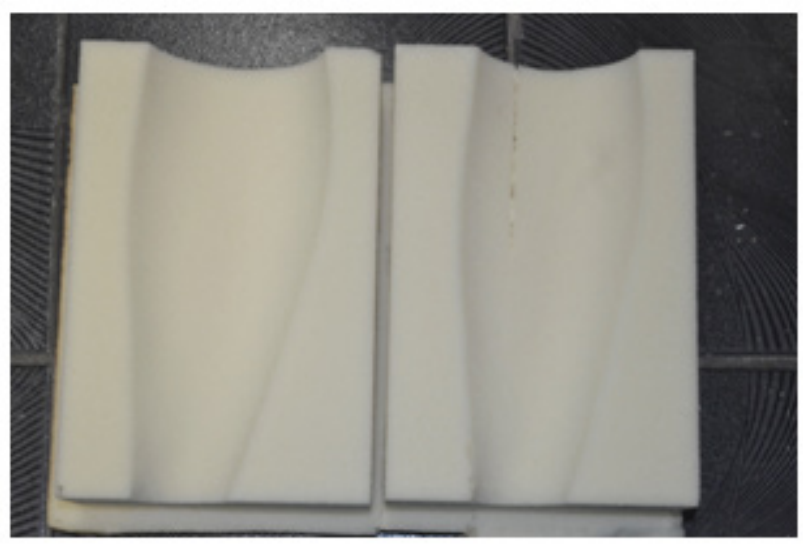

Figura 08: usinagem - acabamento

\section{TÉCNICA DE ANÁLISE POR MAPEAMENTO DE PRESSÃO}

A técnica de análise por mapeamento de pressão permite verificar a distribuição da pressão de contato entre o produto e o corpo do usuário. A pressão excessiva em determinados pontos de contato isolados pode acarretar em uma região de desconforto em relação ao uso de um produto e até mesmo em lesões. A distribuição de pressão mais uniforme na região do contato pode ser um indicativo de conforto.

O sistema de mapeamento de pressão utiliza uma espécie de tapete contento uma matriz de sensores que medem individualmente a pressão exercida em cada região de contato entre o produto e corpo do usuário. $O$ tapete possui um formato quadrado de $400 \times 400 \mathrm{~mm}$, contendo 256 sensores distribuídos em uma matriz de 16 x 16 (sensores de $25 \mathrm{~mm}$ ). As avaliações são determinadas por dados numéricos $(\mathrm{mmHg})$ de saída e um mapa codificado com cores representando o percentual da pressão. $O$ tapete contendo os sensores foi acoplado à perna do sujeito digitalizado e, após, os moldes negativos da perna foram pressionados para determinação da pressão (figura 09). 


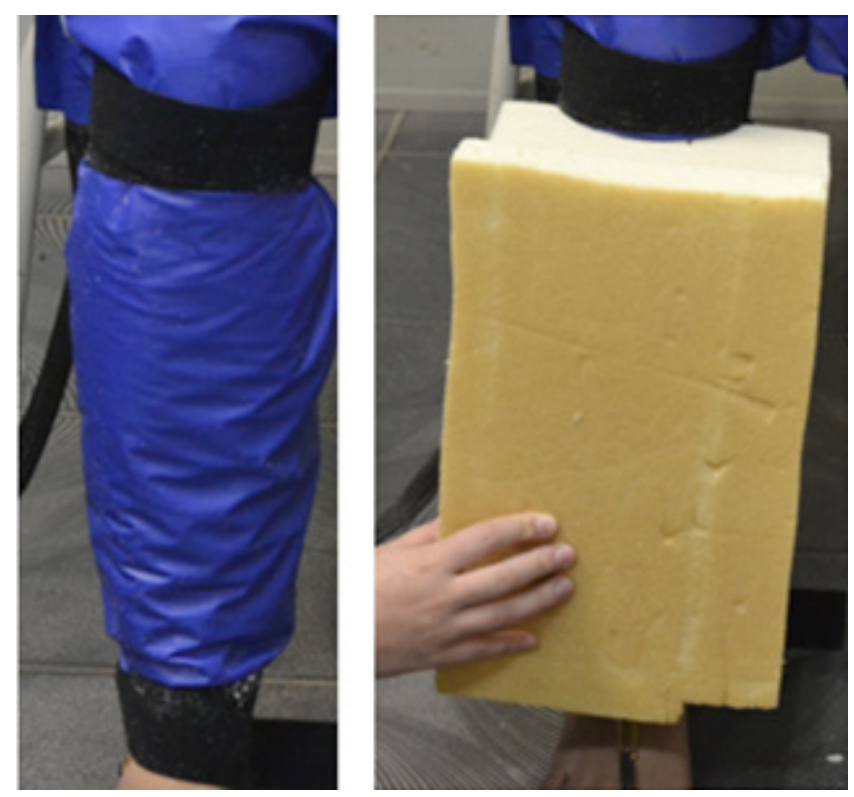

Figura 09: Técnica de análise por mapeamento de pressão

Foi realizado um esforço mínimo de pressão até atingir o pico de um sensor e um esforço suficiente para atingir o maior número de picos nos sensores. Este processo foi efetuado com o molde negativo produzido pelo Artec Eva e pelo Kinect, respectivamente.

\section{TÉCNICA DE ANÁLISE POR TERMOGRAFIA}

A técnica de Termografia complementa a análise por mapeamento de pressão. Através desta análise, é possível medir e identificar a quantidade de energia infravermelha (calor) emitida por uma superfície após o uso de um produto.

Portanto, para esta análise, o sujeito permaneceu na posição sentado e estático por 15 minutos para atingir o equilíbrio térmico com o ambiente. Após este período, a perna do sujeito foi termografada para adquirir o perfil de temperaturas da perna do usuário antes de cada ensaio.

Após, o molde negativo obtido com o scanner 3D Artec Eva foi pressionado diretamente na perna no sujeito por 15 minutos e, imediatamente, a perna termografada. Este processo foi repetido com o molde obtido pelo Kinect após uma nova espera de 15 minutos.

\section{RESULTADOS E DISCUSSÃO}

A primeira análise foi realizada através do software Geomagic Qualify para comparação da diferença dimensional e do volume entre os modelos. Os modelos 3D sobrepostos foram mensurados e o resultado é apresentado através da paleta de cores que vai do verde (dentro de uma precisão de 0 a $1 \mathrm{~mm}$ ) para o azul no sentido negativo e para o vermelho no sentido positivo (precisão de 1 a $7 \mathrm{~mm}$ ). A representação da 
análise de diferença dimensional das malhas 3D segue na figura 10.

Conforme a representação da imagem das cores, é percebido que há poucas regiões que obtiveram uma conformidade das malhas 3D de 100\%, como definido pela cor verde. Na maioria das regiões observa-se a variação dimensional de até $7 \mathrm{~mm}$, principalmente na cor vermelha. Isso indica que a malha obtida pelo scanner Kinect ficou de maior tamanho do que a do Artec Eva.

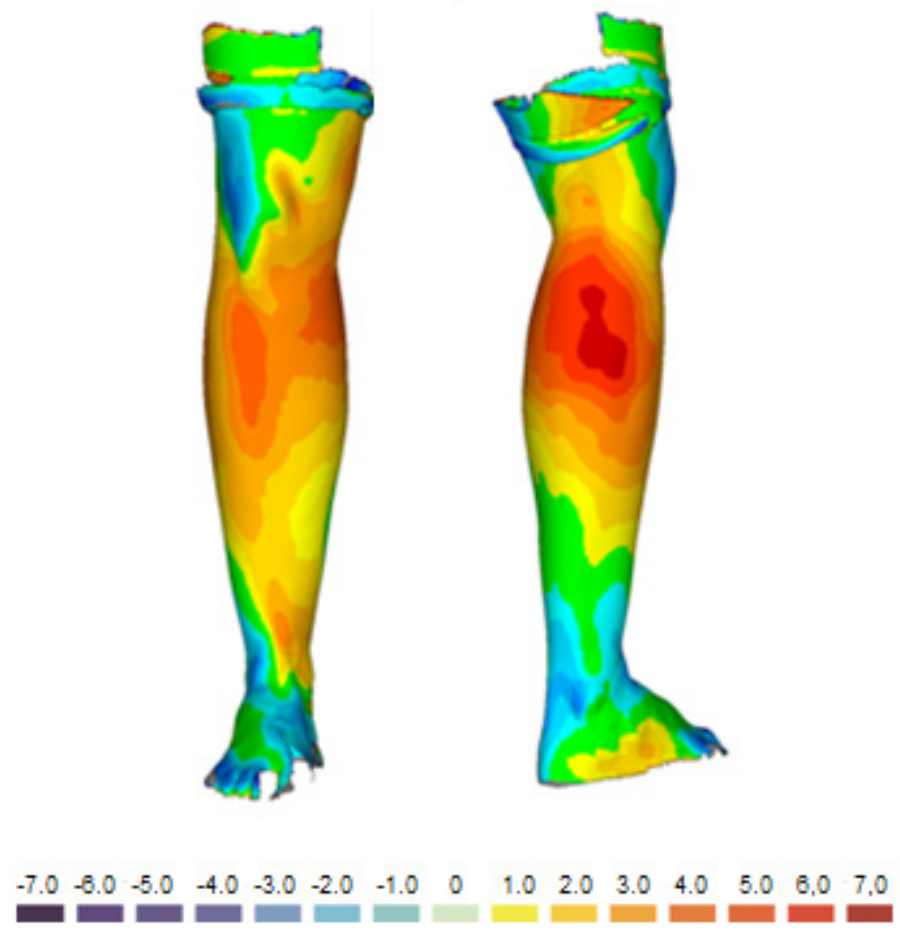

Figura 10: análise no Geomagic Qualify

Para a análise de volume, o modelo 3D obtido pelo scanner Kinect foi diferenciado através da coloração vermelha. Quando sobreposto ao do modelo Artec (azul) fica visível a diferença de volume entre os modelos dos diferentes scanners. A diferença volumétrica medida entre os dois modelos foi de $277.596 \mathrm{~mm} 3$, o que representa cerca de $12 \%$. Esta diferença segue ilustrada na figura 11.

A figura 12 apresenta imagens da análise visual de encaixe do molde negativo usinado sobre a perna do sujeito. Nela fica evidente, pelas folgas observadas, que houve uma diferença dimensional e de precisão entre os modelos.

Observou-se uma precisão maior de medidas antropométricas com o molde negativo sobre o modelo 3D obtido pelo scanner de luz branca, Artec Eva. Por outro, são visíveis as folgas entre a perna do sujeito e o molde desenvolvido com o modelo 3D obtido pelo scanner Kinect. Tal observação condiz com as análises dimensionais e volumétricas apresentadas nas figuras 10 e 11. 


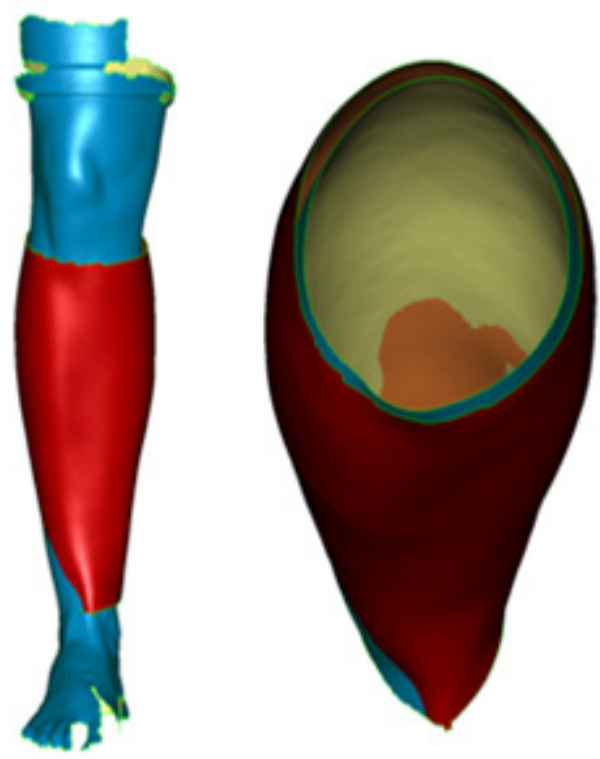

Figura 11: Análise do volume

Portanto, através desta análise, é notório que há uma diferença de precisão das medidas antropométricas e do volume entre os modelos 3D obtidos pelos diferentes scanners.
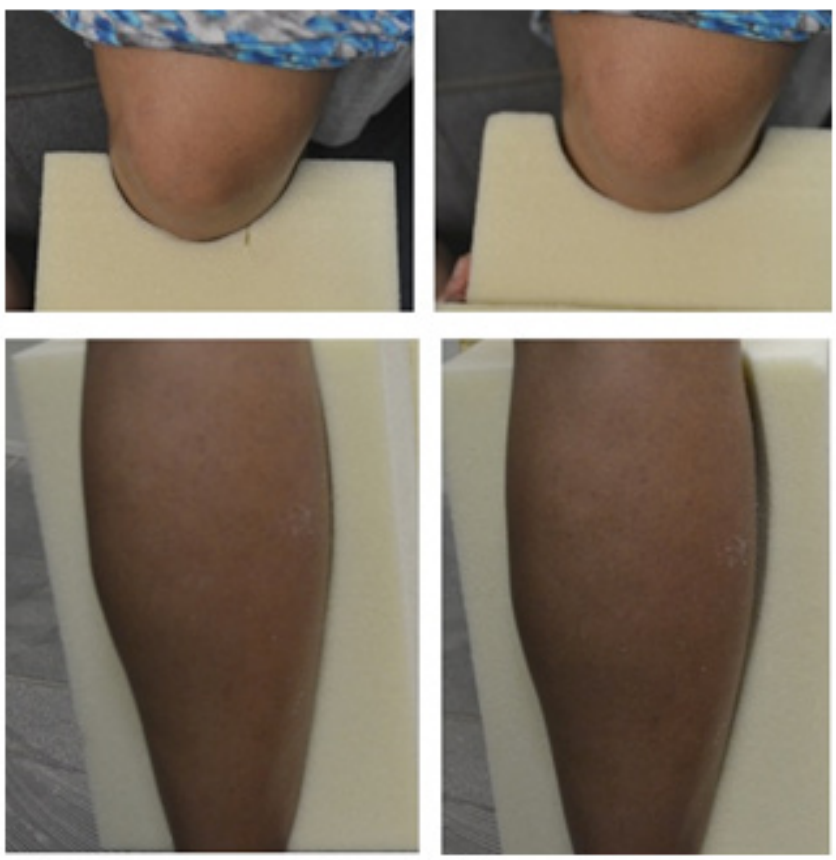

Figura 12: análise visual através do encaixe dos moldes

\section{TÉCNICA DE ANÁLISE POR MAPEAMENTO DE PRESSÃO}

Os resultados da análise por mapeamento de pressão são apresentados nas figuras 13 e 14. A figura 13 (A) e (B) apresentam o mapeamento de pressão com o esforço mínimo para atingir o pico de um sensor, para o molde produzido pelo Artec 
Eva e pelo Kinect, respectivamente. Em ambas figuras é possível observar o maior ponto de pressão localizado na junta, mais ao lado da cabeça da tíbia. Na figura 13 (A) observa-se uma maior área de contato, com pressões mais distribuídas. Na figura 13 (B), aparecem alguns picos menores de pressão em outras partes mais baixas da tíbia.

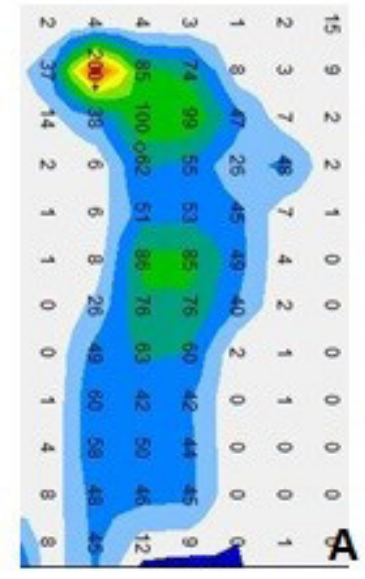

Luz Branca

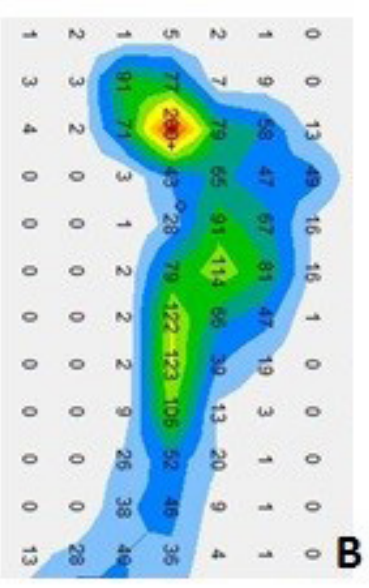

Kinect

Figura 13: análise de mapeamento de pressão com mínimo de esforço.

As figuras 14 (A) e (B) apresentam o mapeamento de pressão com o esforço suficiente para atingir o maior número de picos nos sensores, para o molde produzido pelo Artec e pelo Kinect, respectivamente.

$\mathrm{Na}$ figura 14 (A), observam-se picos em uma linha mais contínua ao longo da tíbia. Já na figura 14 (B), a continuidade é menor, indicando, novamente, uma distribuição menos homogênea da pressão.

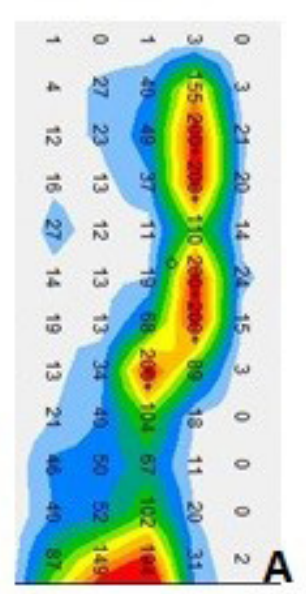

Luz Branca

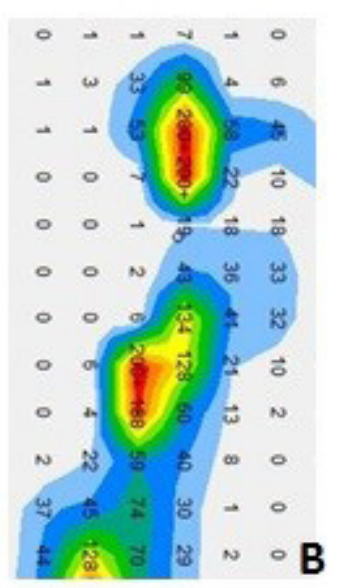

Kinect

Figura 14: análise de mapeamento de pressão com esforço suficiente. 


\section{TÉCNICA DE ANÁLISE POR TERMOGRAFIA}

Na figura 15 (A) é possível observar o perfil de temperaturas da perna do usuário antes de cada ensaio. Tal perfil se repetiu para os dois casos analisados. Nas figuras 15 (B) e (C) observam-se os perfis gerados pelo contato dos moldes produzidos a partir do Artec e do Kinect, respectivamente.

O perfil de temperaturas (B) apresenta um menor aquecimento da região de contato, devido ao respectivo molde possuir uma geometria mais ajustada à perna. Nesse caso, o calor flui da perna para o material do molde predominantemente por condução térmica, porém, como o poliuretano é isolante térmico, no curto prazo, a perna aquece com um perfil de temperaturas mais próximo à sua condição inicial.

Já no perfil (C), devido à falta de contato da perna com partes do molde, cria-se uma região onde o ar fica retido. Nesse caso, acredita-se que o ar aquecido contribui para a distribuição do calor pela superfície da perna. Tal fato pode ser desejado ou não, dependendo da aplicação de um possível produto a ser projetado.

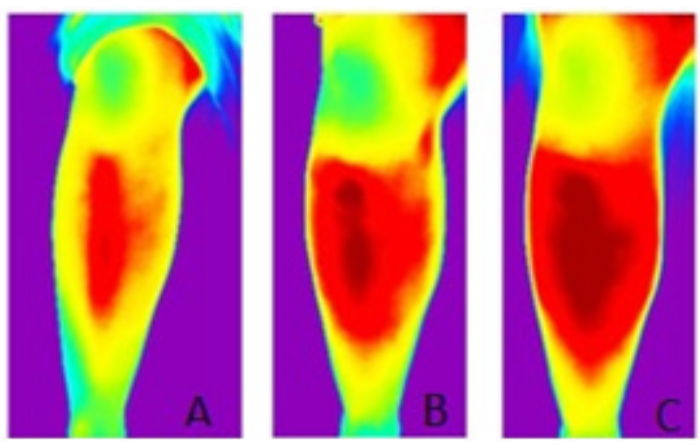

Figura 15: análise por termografia.

\section{CONSIDERAÇÕES FINAIS}

As medidas antropométricas são necessárias para o desenvolvimento de projetos de produtos mais adequados às características físicas do usuário e estão diretamente relacionadas às questões de conforto, assim como o melhor desempenho da função de um produto e suas especificações. Para produtos que exigem personalização de medidas o mais indicado é obter as medidas antropométricas diretamente no usuário final do produto. Através da digitalização tridimensional é possível obter estas medidas antropométricas por meio do modelo 3D do corpo e, assim, medições de forma, silhueta e volume.

O estudo realizado neste artigo, sobre a precisão dimensional, análise de pressão e termografia, nos modelos 3D gerados por diferentes digitalizadores tridimensionais traz importante contribuição para área do Design, no desenvolvimento de produtos, principalmente, em projetos de produtos personalizados, uma vez que viabiliza o uso destes diferentes scanners 3D como uma ferramenta de projeto.

Os resultados obtidos nas análises de precisão dimensional e volume determinaram que há diferença de precisão entre os modelos 3D obtidos pelos scanners, demonstrando que o Artec Eva é mais preciso em relação ao Kinect. No mesmo sen- 
tido, as análises de mapeamento de pressão e termografia revelaram uma maior área de contato do usuário com o molde usinado obtido pelo Artec Eva. Já, nas análises realizadas com o molde obtido pelo Kinect, houve uma distribuição menos homogênea de pressão, o que potencialmente poderia gerar regiões de desconforto no uso do produto.

Entretanto, estes resultados não invalidam o uso do scanner Kinect no desenvolvimento de projetos de produto. O que irá determinar qual a ferramenta mais adequada ao projeto é a exigência da necessidade de precisão no produto. Por exemplo, há diferentes necessidades de precisão para projetos de calçados, vestuário, joias ou Tecnologia Assistiva. Neste contexto, há poucos trabalhos desenvolvidos que relacionam a necessidade da precisão de medidas antropométricas ao projeto de produtos personalizados. A continuidade desta pesquisa buscará investigar a relação entre a precisão de modelos 3D obtidos por digitalização e suas consequências para o produto final.

\section{REFERÊNCIAS}

AITPAYEV, K.; GABER, J. Creation of 3D Human Avatar using Kinect. Asian Transactions on Fundamentals of Electronics, Communication \& Multimedia. 1 (5), p.12-24, 2012.

BRENDLER, C., 2013, Método para levantamento de parâmetros antropométricos utilizando um digitalizador 3D de baixo custo. UFRGS, Porto Alegre, p. 148, 2013. (Dissertação de Mestrado em Design- PgDesign da Universidade Federal do Rio Grande do Sul).

CLARKSON, J. Human capability and product design. In: SCHIFFERSTEIN, H.; HEKKERT, P. (Org.). Product Experience. Elsevier, San Diego. Product Experience, p. 165-198, 2008.

D'APUZZO, N. Recent Advances in 3D Full Body scanning with applications to fashion and apparel. In optical 3D measurement techniques, 9, Viena, Austria, Anais eletrônicos, 2009.

DUTTA, T. Evaluation of the Kinect sensor for 3-D kinematic measurement in the workplace. Applied ergonomics, 43 (4), p. 645-649, 2012.

FOURIE, Z; DAMSTRA, J; GERRITS, P. O.; REN. Y. Evaluation of anthropometric accuracy and reliability using different three-dimensional scanning systems. Forensic Science International, 207, p. 127-134, 2011.

GONZALEZ, J. H; RIVEIRO, B.; FERNANDEZ, V.; SÁNCHEZ, M.; ARIAS, P. Metrological evaluation of Microsoft Kinect and Asus Xtion sensors. Measurement, 46, $p$. 1800-1806, 2013. 
HAN, H.; NAM, Y.; CHOI, K. Comparative analysis of 3D body scan measurements and manual measurements of size Korea adult females. International Journal of Industrial Ergonomics, 40 (5), p. 530-540, 2010.

HERSH, M. The Design and Evaluation of Assistive Technology Products and Devices Part 1: Design. In: STONE H.; BLOUIN, M. (Org.). International Encyclopedia of rehabilitation, 2010.

IIDA, I. Ergonomia: projeto e produção. Edgard Blücher, São Paulo, p. 614, 2005.

LI, Z; CHANG, C.; DEMPSEY, P. G.; CAI, X. Refraction effect analysis of using a hand -held laser scanner with glass support for 3D anthropometric measurement of the hand: A theoretical study. Measurement, 41 (8), p. 842-850, 2008.

LÖBACH, B. Design industrial: Bases para a configuração dos produtos industriais. Blücher, São Paulo, p. 206, 2000.

LU, J.; WANG, M.; MOLLARD. The effect of arm posture on the scan-derived measurements. Applied Ergonomics, 41 (2), p. 236-241, 2010.

LU, J.; WANG, M. J. Automated anthropometric data collection using 3D whole body scanners. Expert Systems with Applications, 35 (1-2), p. 407-414, 2008.

OZSOY, U.; DEMIREL, B.; YILDIRIM, F.; TOSUN, O.; SARIKCIOGLU, L. Method selection in craniofacial measurements: Advantages and disadvantages of 3D digitization method. Journal of Cranio-Maxillofacial Surgery, 37, p. 285-290, 2009.

PANERO, J.; ZELNIK, M. Las dimensiones humanas en los espacios interiores: estándares antropométricos. G. Gill, México, p. 314, 2002.

PASCHOARELLI, L. O posto de trabalho carteira escolar como objeto de desenvolvimento da educação infantil: uma contribuição do design e da ergonomia. UNESP, Bauru, pp. 121, 1997. (Dissertação de Mestrado em Desenho Industrial da Faculdade de Arquitetura, Artes e Comunicação da Universidade Estadual Paulista, Campus Bauru).

PEQUINI, S. Ergonomia aplicada ao design de produtos: um estudo de caso sobre o design de bicicletas. USP, São Paulo, p. 675, 2005. (Tese de doutorado apresentada à Faculdade de Arquitetura e urbanismo da Universidade de São Paulo, Departamento de Tecnologia).

SILVA, F. Usinagem de Espumas de Poliuretano e Digitalização Tridimensional para Fabricação de Assentos Personalizados para Pessoas com Deficiência. UFRGS, Porto Alegre, p. 192, 2011. (Tese de Doutorado em Engenharia de Minas, Metalurgia e de Materiais - PPGE3M da Universidade Federal do Rio Grande do Sul). 
SIMMONS, Karla Peavy. Body measurement techniques: a comparison of threedimensional body scanning and physical anthropometric methods. Raleigh: NCSU, p.68, 2001. (Tese de Doutorado. North Carolina State University).

SCHOENARDIE, R.; TEIXEIRA, C.; MERINO, E. Design e Antropometria: diferenciação estratégica. Projética, 2 (2), p. 31-42, 2011.

TOMKINSON. G, SHAW, L. Quantification of the postural and technical errors in asymptomatic adults using direct 3D whole body scan measurements of standing posture. Gait \& Posture, 37, p. 172-177, 2013.

TONG, J; ZHOU, J; LIU, L; PAN, Z; YAN, H., 2012, "Scanningn 3D full human bodies using kinects". IEEE Transactions on Visualization and Computer Graphics, 18 (4), p. $643-650,2012$.

ULRICH K.; EPPINGER S. Product design and development. McGraw-Hill Higher Education, New York, p. 368, 2008.

WANG, M.; WU, W.; LIN, K.; YANG, S.; LU, J. Automated anthropometric data collection from three-dimensional digital human models". The International Journal of Advanced Manufacturing Technology, 32(1-2), p. 109-115, 2007.

WANG, M.; WU, F.; MA, M.; CHANG, R. A new user-centered design approach: A hair washing assistive device design for users with shoulder mobility restriction. Applied Ergonomics, 40 (5), p. 878-886, 2009. 